\title{
Experimental and DFT Studies on Competitive Heterocyclic Rearrangements. Part 2: A One- atom Side-chain versus the Classic Three-atom Side-chain (Boulton-Katritzky) Ring Rearrangement of 3-Acylamino-1,2,4-oxadiazoles.
}

Andrea Pace, ${ }^{*}{ }^{\dagger}$ Ivana Pibiri,,$^{\dagger}$ Antonio Palumbo Piccionello, ${ }^{\dagger}$ Silvestre Buscemi,${ }^{\dagger}$ Nicolò Vivona, ${ }^{\dagger}$ and

$$
\text { Giampaolo Barone* }{ }^{\Psi}
$$

Dipartimento di Chimica Organica "E. Paternò"and Dipartimento di Chimica Inorganica e Analitica "S.

Cannizzaro" - Università degli Studi di Palermo - Viale delle Scienze - Parco d'Orleans II - Edificio

$$
17 \text { - I-90128 - Palermo - Italy }
$$

\section{pace@unipa.it; gbarone@unipa.it}

\section{Supporting Information Available.}

Cartesian coordinates, B3LYP energy (in a.u.) and standard thermochemical data (in a.u.) at $298.15 \mathrm{~K}$ of compounds reported in Table 1 of the main text.

Neutral Species

Anionic Deprotonated Forms

Transition States

Cartesian coordinates, B3LYP energy (in a.u.) and standard thermochemical data (in a.u.) at

$298.15 \mathrm{~K}$ of compounds reported in Table 2 of the main text.

Neutral Species

Anionic Deprotonated Forms

Transition States

Spectrum of Compound 29b in DMSO- $d_{6}$

NMR Spectrum of Compound 29d in DMSO- $d_{6}$

NMR Spectrum of Compound 30a in DMSO- $d_{6}$

NMR Spectrum of Compound 30b in DMSO- $d_{6}$ 
Cartesian coordinates, B3LYP energy (in a.u.) and standard thermochemical data (in a.u.) at 298.15 $\mathrm{K}$ of compounds reported in Table 1 of the main text.

Compound 29a

\section{Neutral Species}

B3LYP Energy=-509.4622637

Sum of electronic and zero-point Energies $=-509.333883$

Sum of electronic and thermal Enthalpies $=-509.322887$

Sum of electronic and thermal Free Energies $=-509.371383$

Cartesian coordinates

$\begin{array}{cccc}\mathrm{O} & 1.611464 & 1.202468 & 0.866777 \\ \mathrm{~N} & 0.239242 & 1.132833 & 0.495203 \\ \mathrm{C} & 0.160798 & -0.082904 & 0.003126 \\ \mathrm{~N} & 1.333664 & -0.811206 & 0.014371 \\ \mathrm{C} & 2.173251 & 0.028064 & 0.551396 \\ \mathrm{~N} & -0.970364 & -0.688548 & -0.516398 \\ \mathrm{C} & 3.619230 & -0.166059 & 0.839153 \\ \mathrm{H} & 3.919742 & -1.164490 & 0.520819 \\ \mathrm{H} & 3.814430 & -0.051843 & 1.909922 \\ \mathrm{H} & 4.217972 & 0.580578 & 0.308468 \\ \mathrm{H} & -0.808385 & -1.632441 & -0.839006 \\ \mathrm{C} & -2.247646 & -0.145420 & -0.623366 \\ \mathrm{O} & -2.513500 & 0.984079 & -0.260636 \\ \mathrm{C} & -3.281962 & -1.089290 & -1.212539 \\ \mathrm{H} & -2.856410 & -1.992173 & -1.659260 \\ \mathrm{H} & -3.977372 & -1.382946 & -0.419851 \\ \mathrm{H} & -3.853506 & -0.546968 & -1.969073\end{array}$

Compound 30a

B3LYP Energy=-509.4701259

Sum of electronic and zero-point Energies $=-509.341703$

Sum of electronic and thermal Enthalpies $=-509.330756$

Sum of electronic and thermal Free Energies $=-509.379275$

Cartesian coordinates

$\begin{array}{rrrr}\mathrm{C} & 1.959737 & 0.711908 & -0.371693 \\ \mathrm{~N} & 1.494327 & 1.835069 & -0.811011 \\ \mathrm{~N} & 0.098626 & 1.763559 & -0.719238 \\ \mathrm{C} & -0.152539 & 0.587873 & -0.226911 \\ \mathrm{O} & 0.951599 & -0.145243 & 0.023983 \\ \mathrm{~N} & -1.416080 & 0.097983 & 0.026793 \\ \mathrm{C} & 3.364217 & 0.253322 & -0.245838 \\ \mathrm{H} & 4.023154 & 1.046789 & -0.601114 \\ \mathrm{H} & 3.534996 & -0.650099 & -0.840251 \\ \mathrm{H} & 3.607860 & 0.022143 & 0.796210 \\ \mathrm{H} & -2.144990 & 0.753333 & -0.223098 \\ \mathrm{C} & -1.775098 & -1.142807 & 0.554954 \\ \mathrm{O} & -0.958976 & -1.979568 & 0.891675 \\ \mathrm{C} & -3.274148 & -1.363239 & 0.639327\end{array}$




$$
\begin{array}{lrrr}
H & -3.851045 & -0.434774 & 0.677380 \\
H & -3.596617 & -1.936686 & -0.236712 \\
H & -3.485467 & -1.960826 & 1.527470
\end{array}
$$

Compound 29a $\mathbf{a}^{-}$

\section{Deprotonated Anionic Forms}

B3LYP Energy=-508.9042038

Sum of electronic and zero-point Energies $=-508.790177$

Sum of electronic and thermal Enthalpies $=-508.779409$

Sum of electronic and thermal Free Energies $=-508.827290$

Cartesian coordinates

$\begin{array}{lrrr}\mathrm{N} & -1.204450 & -1.007248 & 0.000023 \\ \mathrm{C} & -0.073055 & -0.153087 & -0.000012 \\ \mathrm{~N} & -0.407263 & 1.143166 & -0.000050 \\ \mathrm{O} & -1.864438 & 1.106188 & -0.000037 \\ \mathrm{C} & -2.203066 & -0.188843 & 0.000011 \\ \mathrm{~N} & 1.151435 & -0.729816 & -0.000053 \\ \mathrm{C} & -3.658954 & -0.525198 & 0.000033 \\ \mathrm{H} & -4.155376 & -0.108762 & 0.884089 \\ \mathrm{H} & -3.776890 & -1.610232 & 0.000349 \\ \mathrm{H} & -4.155287 & -0.109261 & -0.884315 \\ \mathrm{C} & 2.273896 & 0.022899 & -0.000009 \\ \mathrm{O} & 2.394263 & 1.265364 & 0.000077 \\ \mathrm{C} & 3.561085 & -0.818395 & -0.000012 \\ \mathrm{H} & 3.350130 & -1.890934 & -0.000545 \\ \mathrm{H} & 4.160944 & -0.559691 & -0.881520 \\ \mathrm{H} & 4.160394 & -0.560504 & 0.882107\end{array}$

Compound $\mathbf{3 0 a}^{-}$

B3LYP Energy=-508.9221077

Sum of electronic and zero-point Energies $=-508.807875$

Sum of electronic and thermal Enthalpies $=-508.797190$

Sum of electronic and thermal Free Energies=-508.845673

Cartesian coordinates
O $\quad 0.984654-1.089299$
0.216359
$\begin{array}{llll}\mathrm{C} & 0.065593 & -0.035970 & 0.005843\end{array}$
$\mathrm{N} \quad 0.725721 \quad 1.076407 \quad-0.277624$
N $\quad 2.092661 \quad 0.761702 \quad-0.255949$
$\begin{array}{llll}\text { C } & 2.189712 & -0.496177 & 0.033054\end{array}$
$\mathrm{N} \quad-1.210950 \quad-0.407140 \quad 0.140133$
$\begin{array}{llll}\text { C } & 3.425364 & -1.312289 & 0.175999\end{array}$
$\mathrm{H} \quad 4.291579 \quad-0.670816 \quad-0.003041$
H $\quad 3.443722 \quad-2.142323 \quad-0.541969$
H $\quad 3.506062-1.745875 \quad 1.181015$
$\begin{array}{llll}\text { C } & -2.230228 & 0.471769 & -0.025806\end{array}$
$\begin{array}{llll}\text { O } & -2.191006 & 1.682736 & -0.308413\end{array}$
$\begin{array}{llll}\text { C } & -3.610635 & -0.173572 & 0.176154\end{array}$
$\mathrm{H} \quad-3.536897 \quad-1.236770 \quad 0.419230$ 


$$
\begin{array}{cccc}
\mathrm{H} & -4.139312 & 0.352870 & 0.980373 \\
\mathrm{H} & -4.205176 & -0.043921 & -0.736555
\end{array}
$$

Compound $\mathbf{3 1}=\mathbf{3 1}$,

B3LYP Energy=-508.8708646

Sum of electronic and zero-point Energies $=-508.759767$

Sum of electronic and thermal Enthalpies $=-508.747833$

Sum of electronic and thermal Free Energies $=-508.799429$

Cartesian coordinates

$\begin{array}{lccc}\mathrm{C} & -3.129760 & 1.443508 & 0.612932 \\ \mathrm{C} & -1.603899 & 1.539147 & 0.550400 \\ \mathrm{O} & -1.054192 & 2.649973 & 0.556959 \\ \mathrm{~N} & -1.004463 & 0.308910 & 0.494668 \\ \mathrm{C} & 0.312088 & 0.274110 & 0.399782 \\ \mathrm{~N} & 1.243821 & -0.820687 & 0.409113 \\ \mathrm{C} & 1.471144 & -1.564896 & -0.753009 \\ \mathrm{O} & 2.132589 & -1.217998 & -1.722873 \\ \mathrm{~N} & 1.465685 & 0.790400 & 0.254490 \\ \mathrm{C} & 0.895495 & -2.972537 & -0.626240 \\ \mathrm{H} & -3.436926 & 0.807869 & 1.450895 \\ \mathrm{H} & -3.511586 & 0.974152 & -0.301495 \\ \mathrm{H} & -3.561569 & 2.441166 & 0.721072 \\ \mathrm{H} & -0.143750 & -2.925339 & -0.284223 \\ \mathrm{H} & 0.959884 & -3.480172 & -1.591473 \\ \mathrm{H} & 1.461065 & -3.539823 & 0.121446\end{array}$

Compound 31"

B3LYP Energy $=-508.8733782$

Sum of electronic and zero-point Energies $=-508.761956$

Sum of electronic and thermal Enthalpies $=-508.750286$

Sum of electronic and thermal Free Energies $=-508.800411$

Cartesian coordinates

$\begin{array}{lrrr}\mathrm{C} & 3.388571 & -0.466916 & -0.133498 \\ \mathrm{C} & 1.864626 & -0.363580 & -0.057115 \\ \mathrm{~N} & 1.424425 & 0.889244 & 0.233497 \\ \mathrm{C} & 0.139123 & 1.184682 & 0.139658 \\ \mathrm{~N} & -0.751495 & 2.042799 & 0.415257 \\ \mathrm{~N} & -1.074822 & 0.755114 & -0.526883 \\ \mathrm{C} & -1.917102 & -0.193974 & 0.085945 \\ \mathrm{C} & -2.027833 & -1.456515 & -0.752045 \\ \mathrm{O} & 1.187803 & -1.390676 & -0.253622 \\ \mathrm{O} & -2.564782 & -0.016773 & 1.108849 \\ \mathrm{H} & 3.876549 & 0.383095 & 0.349476 \\ \mathrm{H} & 3.687750 & -0.487204 & -1.189380 \\ \mathrm{H} & 3.717017 & -1.407372 & 0.319930 \\ \mathrm{H} & -1.028028 & -1.889013 & -0.858784 \\ \mathrm{H} & -2.706011 & -2.158598 & -0.261357 \\ \mathrm{H} & -2.402509 & -1.213592 & -1.752464\end{array}$


Compound 32

B3LYP Energy=-508.9030411

Sum of electronic and zero-point Energies $=-508.791162$

Sum of electronic and thermal Enthalpies $=-508.779017$

Sum of electronic and thermal Free Energies $=-508.831571$

Cartesian coordinates

$\begin{array}{lrrr}\mathrm{C} & -4.188838 & -0.498033 & -0.125068 \\ \mathrm{H} & -4.159358 & -1.250254 & -0.916578 \\ \mathrm{C} & -2.825602 & 0.150918 & 0.095233 \\ \mathrm{O} & -2.739487 & 1.189080 & 0.763863 \\ \mathrm{~N} & -1.798457 & -0.536838 & -0.497207 \\ \mathrm{C} & -0.574403 & -0.130974 & -0.345466 \\ \mathrm{~N} & 0.585988 & 0.032456 & -0.288876 \\ \mathrm{~N} & 1.800351 & 0.577048 & -0.489935 \\ \mathrm{H} & -4.926380 & 0.275375 & -0.360347 \\ \mathrm{H} & -4.504735 & -0.979947 & 0.808298 \\ \mathrm{C} & 2.794393 & -0.130161 & 0.105905 \\ \mathrm{O} & 2.707447 & -1.176758 & 0.776995 \\ \mathrm{C} & 4.162010 & 0.508474 & -0.130695 \\ \mathrm{H} & 4.094180 & 1.412651 & -0.741311 \\ \mathrm{H} & 4.614002 & 0.753033 & 0.837499 \\ \mathrm{H} & 4.818075 & -0.219435 & -0.621755\end{array}$

Transition States

Transition State $\left[\mathbf{2 9} \mathbf{a}^{-} \rightarrow \mathbf{2 9} \mathbf{a}^{{ }^{-}}\right]^{\ddagger}$

B3LYP Energy=-508.8807012

Sum of electronic and zero-point Energies $=-508.767842$

Sum of electronic and thermal Enthalpies $=-508.757687$

Sum of electronic and thermal Free Energies $=-508.802826$

Cartesian coordinates

$\begin{array}{lrrr}\mathrm{N} & 1.184438 & -0.935968 & 0.000060 \\ \mathrm{C} & 0.000043 & -0.211126 & 0.000007 \\ \mathrm{~N} & 0.000176 & 1.123132 & -0.000110 \\ \mathrm{O} & 1.993744 & 1.182753 & -0.000101 \\ \mathrm{C} & 2.188648 & -0.078053 & -0.000008 \\ \mathrm{~N} & -1.184397 & -0.935833 & 0.000070 \\ \mathrm{C} & 3.612054 & -0.599302 & 0.000076 \\ \mathrm{H} & 4.144203 & -0.226375 & -0.882556 \\ \mathrm{H} & 3.623100 & -1.691280 & -0.000019 \\ \mathrm{H} & 4.144022 & -0.226559 & 0.882899 \\ \mathrm{C} & -2.188718 & -0.077961 & 0.000004 \\ \mathrm{O} & -1.993877 & 1.182804 & -0.000090 \\ \mathrm{C} & -3.612089 & -0.599388 & 0.000061 \\ \mathrm{H} & -3.622965 & -1.691363 & 0.000108 \\ \mathrm{H} & -4.144200 & -0.226573 & 0.882769 \\ \mathrm{H} & -4.144239 & -0.226651 & -0.882656\end{array}$




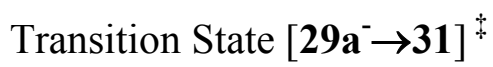

B3LYP Energy $=-508.8614812$

Sum of electronic and zero-point Energies $=-508.750615$

Sum of electronic and thermal Enthalpies $=-508.739422$

Sum of electronic and thermal Free Energies=-508.788436

Cartesian coordinates

$\begin{array}{lrrr}\mathrm{N} & -1.277993 & 0.197670 & -0.775589 \\ \mathrm{C} & 0.072780 & 0.248846 & -0.371608 \\ \mathrm{~N} & 0.043670 & 1.481511 & -0.072557 \\ \mathrm{O} & -2.008575 & -0.302866 & 1.406624 \\ \mathrm{C} & -2.193898 & -0.162609 & 0.195445 \\ \mathrm{~N} & 1.038639 & -0.692165 & -0.396150 \\ \mathrm{C} & -3.580073 & -0.392146 & -0.408885 \\ \mathrm{H} & -4.244368 & -0.820578 & 0.345056 \\ \mathrm{H} & -3.518327 & -1.052633 & -1.279946 \\ \mathrm{H} & -3.986536 & 0.564570 & -0.757139 \\ \mathrm{C} & 2.192592 & -0.105689 & -0.012345 \\ \mathrm{O} & 2.305868 & 1.106816 & 0.306711 \\ \mathrm{C} & 3.428029 & -0.994334 & 0.025425 \\ \mathrm{H} & 3.192304 & -2.009555 & -0.300745 \\ \mathrm{H} & 4.205608 & -0.567789 & -0.618111 \\ \mathrm{H} & 3.826179 & -1.019139 & 1.046091\end{array}$

Transition State $\left[\mathbf{2 9 a ^ { - } \rightarrow \mathbf { 3 1 }}{ }^{\prime}\right]^{*}$

B3LYP Energy $=-508.8383864$

Sum of electronic and zero-point Energies $=-508.728906$

Sum of electronic and thermal Enthalpies $=-508.717629$

Sum of electronic and thermal Free Energies=-508.766322

Cartesian coordinates

$\begin{array}{lrrr}\mathrm{N} & -1.176370 & -0.575552 & -0.283440 \\ \mathrm{C} & 0.118923 & -0.003750 & -0.144404 \\ \mathrm{~N} & -0.048418 & 1.254457 & -0.173803 \\ \mathrm{O} & -2.549642 & 1.236438 & 0.226605 \\ \mathrm{C} & -2.323024 & 0.025174 & 0.014370 \\ \mathrm{~N} & 1.168886 & -0.843922 & -0.059790 \\ \mathrm{C} & -3.503866 & -0.959711 & 0.078743 \\ \mathrm{H} & -3.936766 & -0.910040 & 1.083182 \\ \mathrm{H} & -3.208648 & -1.986570 & -0.142764 \\ \mathrm{H} & -4.271465 & -0.633797 & -0.630623 \\ \mathrm{C} & 2.441278 & -0.356789 & 0.049859 \\ \mathrm{O} & 3.412447 & -1.120584 & 0.172007 \\ \mathrm{C} & 2.703737 & 1.155965 & 0.017920 \\ \mathrm{H} & 2.121731 & 1.668846 & 0.794437 \\ \mathrm{H} & 3.769147 & 1.344668 & 0.171726 \\ \mathrm{H} & 2.392586 & 1.579850 & -0.944554\end{array}$


Transition State $\left[\mathbf{3 1} \rightarrow \mathbf{3 1}{ }^{\prime}\right]^{*}$

B3LYP Energy $=-508.8377862$

Sum of electronic and zero-point Energies $=-508.728640$

Sum of electronic and thermal Enthalpies $=-508.716689$

Sum of electronic and thermal Free Energies $=-508.769141$

Cartesian coordinates

$\begin{array}{lrrr}\mathrm{N} & 1.056191 & 0.038163 & 0.607271 \\ \mathrm{C} & 0.000162 & 0.684209 & 0.001230 \\ \mathrm{~N} & 0.000203 & 1.937401 & 0.004427 \\ \mathrm{O} & 2.609115 & 0.375781 & -1.118547 \\ \mathrm{C} & 2.234536 & -0.147123 & -0.059508 \\ \mathrm{~N} & -1.056456 & 0.042270 & -0.608444 \\ \mathrm{C} & 3.145476 & -1.162188 & 0.637552 \\ \mathrm{H} & 3.044064 & -2.129932 & 0.131240 \\ \mathrm{H} & 2.879016 & -1.290607 & 1.689221 \\ \mathrm{H} & 4.187203 & -0.841674 & 0.540292 \\ \mathrm{C} & -2.233452 & -0.148168 & 0.059279 \\ \mathrm{O} & -2.604817 & 0.365047 & 1.124220 \\ \mathrm{C} & -3.149126 & -1.153723 & -0.645360 \\ \mathrm{H} & -3.079672 & -2.116128 & -0.123890 \\ \mathrm{H} & -4.186046 & -0.810860 & -0.574517 \\ \mathrm{H} & -2.864098 & -1.300299 & -1.689675\end{array}$

Transition State $\left[\mathbf{3 1}{ }^{\prime \prime} \rightarrow \mathbf{3 2}\right]^{\ddagger}$

B3LYP Energy $=-508.8616796$

Sum of electronic and zero-point Energies $=-508.751995$

Sum of electronic and thermal Enthalpies $=-508.740341$

Sum of electronic and thermal Free Energies $=-508.790391$

Cartesian coordinates

$\begin{array}{rrrr}\mathrm{N} & 1.516671 & 0.870743 & -0.222347 \\ \mathrm{C} & 0.288647 & 1.187035 & -0.071562 \\ \mathrm{~N} & -0.739989 & 1.847451 & 0.095263 \\ \mathrm{O} & 1.456691 & -1.340870 & 0.512217 \\ \mathrm{C} & 2.060231 & -0.350675 & 0.098124 \\ \mathrm{~N} & -1.308514 & 0.641734 & -0.747987 \\ \mathrm{C} & 3.563872 & -0.397955 & -0.153753 \\ \mathrm{H} & 4.058633 & 0.467771 & 0.298849 \\ \mathrm{H} & 3.757066 & -0.353954 & -1.232188 \\ \mathrm{H} & 3.976524 & -1.324513 & 0.250409 \\ \mathrm{C} & -2.054668 & -0.167182 & 0.066064 \\ \mathrm{O} & -2.309660 & -0.047069 & 1.272299 \\ \mathrm{C} & -2.659554 & -1.328188 & -0.731346 \\ \mathrm{H} & -2.404958 & -1.279219 & -1.793145 \\ \mathrm{H} & -2.283269 & -2.266759 & -0.309975 \\ \mathrm{H} & -3.748591 & -1.317523 & -0.609733\end{array}$

Transition State $[\mathbf{3 2} \rightarrow \mathbf{3 0 a}]^{*}$ 
B3LYP Energy=-508.8993171

Sum of electronic and zero-point Energies $=-508.787391$

Sum of electronic and thermal Enthalpies $=-508.776531$

Sum of electronic and thermal Free Energies $=-508.824172$

Cartesian coordinates

$\begin{array}{lrrr}\mathrm{N} & -1.395721 & 0.369665 & -0.688821 \\ \mathrm{C} & -0.225019 & 0.693974 & -0.285620 \\ \mathrm{~N} & 0.734124 & 1.412176 & -0.038984 \\ \mathrm{O} & -2.250308 & -0.170933 & 1.422380 \\ \mathrm{C} & -2.351390 & -0.091892 & 0.197848 \\ \mathrm{~N} & 2.069086 & 1.073698 & 0.129468 \\ \mathrm{C} & -3.630964 & -0.520853 & -0.510196 \\ \mathrm{H} & -3.421766 & -1.391064 & -1.143122 \\ \mathrm{H} & -3.986716 & 0.277421 & -1.170361 \\ \mathrm{H} & -4.397771 & -0.775612 & 0.224290 \\ \mathrm{C} & 2.212737 & -0.250036 & -0.041955 \\ \mathrm{O} & 1.276847 & -1.049210 & -0.319839 \\ \mathrm{C} & 3.630225 & -0.766243 & 0.130529 \\ \mathrm{H} & 4.319166 & 0.051343 & 0.358372 \\ \mathrm{H} & 3.656121 & -1.504365 & 0.940876 \\ \mathrm{H} & 3.952694 & -1.275055 & -0.785661\end{array}$

Cartesian coordinates, B3LYP energy (in a.u.) and standard thermochemical data (in a.u.) at 298.15 $\mathrm{K}$ of compounds reported in Table 2 of the main text.

\section{Neutral Species}

Compound 33

B3LYP Energy=-701.2123702

Sum of electronic and zero-point Energies $=-701.030576$

Sum of electronic and thermal Enthalpies $=-701.016765$

Sum of electronic and thermal Free Energies $=-701.072215$

Cartesian coordinates

$\begin{array}{lrrc}\mathrm{C} & -2.853106 & 1.218602 & -0.023729 \\ \mathrm{C} & -2.029967 & 0.080429 & -0.003506 \\ \mathrm{C} & -2.606103 & -1.200756 & 0.011193 \\ \mathrm{C} & -3.992547 & -1.338661 & 0.005751 \\ \mathrm{C} & -4.811562 & -0.204623 & -0.014404 \\ \mathrm{C} & -4.239520 & 1.071209 & -0.029076 \\ \mathrm{C} & -0.574117 & 0.210829 & 0.002388 \\ \mathrm{O} & -0.026161 & 1.436168 & -0.011058 \\ \mathrm{~N} & 1.384923 & 1.273927 & -0.001715 \\ \mathrm{C} & 1.501312 & -0.036859 & 0.015888 \\ \mathrm{~N} & 0.321400 & -0.74294 & 0.019481 \\ \mathrm{~N} & 2.686529 & -0.752128 & 0.031776 \\ \mathrm{C} & 3.983240 & -0.244345 & 0.019464 \\ \mathrm{O} & 4.221899 & 0.947512 & 0.016503 \\ \mathrm{C} & 5.064696 & -1.310346 & -0.022757\end{array}$




$\begin{array}{lrrr}\mathrm{H} & 2.551161 & -1.753724 & 0.039204 \\ \mathrm{H} & 5.358751 & -1.477440 & -1.064952 \\ \mathrm{H} & 5.936691 & -0.938849 & 0.517413 \\ \mathrm{H} & 4.749667 & -2.266729 & 0.405114 \\ \mathrm{H} & -1.959728 & -2.071661 & 0.026778 \\ \mathrm{H} & -4.435034 & -2.330102 & 0.017203 \\ \mathrm{H} & -5.891921 & -0.315043 & -0.018607 \\ \mathrm{H} & -4.873453 & 1.952457 & -0.044712 \\ \mathrm{H} & -2.405951 & 2.206779 & -0.035062\end{array}$

\section{Compound 34}

B3LYP Energy=-701.2179355

Sum of electronic and zero-point Energies $=-701.035883$

Sum of electronic and thermal Enthalpies $=-701.022233$

Sum of electronic and thermal Free Energies $=-701.077102$ Cartesian coordinates

$\begin{array}{lrrr}\mathrm{C} & 2.381179 & 1.208772 & -0.415192 \\ \mathrm{C} & 1.941931 & -0.117808 & -0.277636 \\ \mathrm{C} & 2.882544 & -1.159034 & -0.282390 \\ \mathrm{C} & 4.242283 & -0.876609 & -0.399305 \\ \mathrm{C} & 4.675692 & 0.447291 & -0.526140 \\ \mathrm{C} & 3.743374 & 1.487964 & -0.540305 \\ \mathrm{C} & 0.498438 & -0.500215 & -0.150967 \\ \mathrm{O} & 0.078146 & -1.609212 & -0.436403 \\ \mathrm{~N} & -0.336278 & 0.515838 & 0.324166 \\ \mathrm{C} & -1.695787 & 0.432343 & 0.534477 \\ \mathrm{O} & -2.458441 & -0.426479 & -0.176046 \\ \mathrm{C} & -3.725367 & -0.131730 & 0.281878 \\ \mathrm{~N} & -3.712972 & 0.812456 & 1.165624 \\ \mathrm{~N} & -2.375605 & 1.188486 & 1.342399 \\ \mathrm{C} & -4.869517 & -0.905079 & -0.258359 \\ \mathrm{H} & -5.786954 & -0.541620 & 0.206605 \\ \mathrm{H} & -4.754232 & -1.972119 & -0.041726 \\ \mathrm{H} & -4.944557 & -0.787511 & -1.344228 \\ \mathrm{H} & 0.083308 & 1.311661 & 0.786447 \\ \mathrm{H} & 2.527416 & -2.180133 & -0.193503 \\ \mathrm{H} & 4.964240 & -1.687629 & -0.393291 \\ \mathrm{H} & 5.735150 & 0.666542 & -0.620460 \\ \mathrm{H} & 4.073865 & 2.515555 & -0.657352 \\ \mathrm{H} & 1.669492 & 2.027960 & -0.464586\end{array}$

Compound 35

B3LYP Energy=-701.2203174

Sum of electronic and zero-point Energies $=-701.038577$

Sum of electronic and thermal Enthalpies $=-701.024697$

Sum of electronic and thermal Free Energies $=-701.080891$

Cartesian coordinates 


$\begin{array}{lrrr}\mathrm{O} & -0.389705 & 0.447630 & -0.003554 \\ \mathrm{C} & -0.249776 & -0.926934 & -0.012569 \\ \mathrm{~N} & -1.402997 & -1.524936 & -0.024164 \\ \mathrm{~N} & -2.380444 & -0.531343 & -0.024446 \\ \mathrm{C} & -1.728126 & 0.594427 & -0.011744 \\ \mathrm{C} & 1.092208 & -1.495598 & -0.008000 \\ \mathrm{~N} & -2.314572 & 1.840797 & -0.004759 \\ \mathrm{C} & -1.691220 & 3.089954 & 0.006085 \\ \mathrm{C} & -2.657411 & 4.257797 & 0.071699 \\ \mathrm{O} & -0.482290 & 3.224774 & -0.009470 \\ \mathrm{H} & -3.325200 & 1.797134 & -0.000876 \\ \mathrm{H} & -3.656855 & 4.019459 & -0.303086 \\ \mathrm{H} & -2.745957 & 4.584891 & 1.113572 \\ \mathrm{H} & -2.239015 & 5.084378 & -0.504726 \\ \mathrm{C} & 2.225694 & -0.666012 & 0.002551 \\ \mathrm{C} & 3.500125 & -1.233634 & 0.006728 \\ \mathrm{C} & 3.653894 & -2.622696 & 0.000489 \\ \mathrm{C} & 2.524153 & -3.448816 & -0.010083 \\ \mathrm{C} & 1.247061 & -2.893125 & -0.014351 \\ \mathrm{H} & 2.104712 & 0.412092 & 0.007034 \\ \mathrm{H} & 4.373395 & -0.588348 & 0.014790 \\ \mathrm{H} & 4.647756 & -3.060274 & 0.003787 \\ \mathrm{H} & 2.639134 & -4.528618 & -0.015008 \\ \mathrm{H} & 0.364459 & -3.523756 & -0.022543\end{array}$

Compound 36

B3LYP Energy=-701.2097501

Sum of electronic and zero-point Energies $=-701.027814$

Sum of electronic and thermal Enthalpies $=-701.014083$

Sum of electronic and thermal Free Energies=-701.069218 Cartesian coordinates

$\begin{array}{lrrr}\mathrm{C} & -2.329110 & -1.177037 & -0.347344 \\ \mathrm{C} & -2.027692 & 0.158380 & -0.036313 \\ \mathrm{C} & -3.066712 & 1.031636 & 0.318928 \\ \mathrm{C} & -4.380855 & 0.571808 & 0.385844 \\ \mathrm{C} & -4.674176 & -0.762145 & 0.084344 \\ \mathrm{C} & -3.647586 & -1.633404 & -0.288674 \\ \mathrm{C} & -0.641087 & 0.733556 & -0.090875 \\ \mathrm{O} & -0.427786 & 1.925615 & -0.231599 \\ \mathrm{~N} & 0.387528 & -0.194602 & 0.043756 \\ \mathrm{C} & 1.746773 & 0.053932 & -0.007324 \\ \mathrm{~N} & 2.322293 & 1.203387 & -0.281004 \\ \mathrm{O} & 3.701114 & 0.857629 & -0.206020 \\ \mathrm{C} & 3.772719 & -0.443862 & 0.102764 \\ \mathrm{~N} & 2.603103 & -1.001161 & 0.239091 \\ \mathrm{C} & 5.116252 & -1.064641 & 0.247723 \\ \mathrm{H} & 5.688528 & -0.958089 & -0.678911 \\ \mathrm{H} & 5.001765 & -2.122773 & 0.483903\end{array}$




$\begin{array}{rrrr}\mathrm{H} & 5.679569 & -0.573221 & 1.047104 \\ \mathrm{H} & 0.163946 & -1.146522 & 0.297596 \\ \mathrm{H} & -2.822079 & 2.065791 & 0.536714 \\ \mathrm{H} & -5.176951 & 1.253344 & 0.670320 \\ \mathrm{H} & -5.698764 & -1.119096 & 0.132607 \\ \mathrm{H} & -3.872080 & -2.665087 & -0.542290 \\ \mathrm{H} & -1.552186 & -1.862998 & -0.673439\end{array}$

Deprotonated Anionic Forms

Compound 33-

B3LYP Energy=-700.6576842

Sum of electronic and zero-point Energies $=-700.490470$

Sum of electronic and thermal Enthalpies $=-700.476803$

Sum of electronic and thermal Free Energies=-700.532350

Cartesian coordinates

$\begin{array}{lrcc}\mathrm{C} & -0.246182 & -0.509392 & 0.000044 \\ \mathrm{~N} & 0.794577 & 0.261528 & 0.000051 \\ \mathrm{C} & 0.249514 & 1.563466 & 0.000075 \\ \mathrm{~N} & -1.094450 & 1.554378 & 0.000020 \\ \mathrm{O} & -1.417162 & 0.142397 & 0.000031 \\ \mathrm{~N} & 1.103656 & 2.609777 & 0.000054 \\ \mathrm{C} & 0.640842 & 3.881326 & 0.000065 \\ \mathrm{C} & 1.763698 & 4.930365 & -0.000266 \\ \mathrm{C} & -0.261899 & -1.977886 & 0.000038 \\ \mathrm{O} & -0.537301 & 4.290699 & -0.000126 \\ \mathrm{H} & 2.755156 & 4.470341 & -0.000663 \\ \mathrm{H} & 1.654867 & 5.573897 & -0.881944 \\ \mathrm{H} & 1.655534 & 5.573699 & 0.881642 \\ \mathrm{C} & -1.470905 & -2.693227 & 0.000031 \\ \mathrm{C} & -1.462364 & -4.089330 & 0.000025 \\ \mathrm{C} & -0.251125 & -4.788454 & 0.000028 \\ \mathrm{C} & 0.955679 & -4.078677 & 0.000035 \\ \mathrm{C} & 0.953238 & -2.684421 & 0.000040 \\ \mathrm{H} & -2.407793 & -2.146014 & 0.000030 \\ \mathrm{H} & -2.404145 & -4.632270 & 0.000020 \\ \mathrm{H} & -0.246918 & -5.875416 & 0.000023 \\ \mathrm{H} & 1.901538 & -4.614504 & 0.000037 \\ \mathrm{H} & 1.878006 & -2.116896 & 0.000046 \\ & & & \end{array}$

Compound 34-

B3LYP Energy=-700.6788335

Sum of electronic and zero-point Energies $=-700.510977$

Sum of electronic and thermal Enthalpies $=-700.497599$

Sum of electronic and thermal Free Energies=-700.551999

Cartesian coordinates
O 2.711637
$0.186556-0.000223$
C $\quad 1.466636 \quad 0.850972 \quad-0.000138$ 


$\begin{array}{lrrr}\mathrm{N} & 1.660459 & 2.159673 & 0.000279 \\ \mathrm{~N} & 3.046428 & 2.369316 & 0.000101 \\ \mathrm{C} & 3.608635 & 1.203083 & -0.000064 \\ \mathrm{~N} & 0.418200 & 0.024587 & -0.000207 \\ \mathrm{C} & -0.852704 & 0.483132 & 0.000147 \\ \mathrm{C} & -1.881959 & -0.643929 & 0.000059 \\ \mathrm{C} & 5.061553 & 0.884255 & -0.000363 \\ \mathrm{O} & -1.271928 & 1.655399 & 0.000567 \\ \mathrm{H} & 5.625414 & 1.819964 & 0.001187 \\ \mathrm{H} & 5.343099 & 0.297878 & 0.883349 \\ \mathrm{H} & 5.343412 & 0.300740 & -0.885889 \\ \mathrm{C} & -3.244859 & -0.312852 & 0.000359 \\ \mathrm{C} & -4.224814 & -1.308915 & 0.000299 \\ \mathrm{C} & -3.855491 & -2.658628 & -0.000059 \\ \mathrm{C} & -2.497530 & -2.999012 & -0.000358 \\ \mathrm{C} & -1.520042 & -2.000129 & -0.000301 \\ \mathrm{H} & -3.504378 & 0.741397 & 0.000641 \\ \mathrm{H} & -5.277595 & -1.033570 & 0.000533 \\ \mathrm{H} & -4.616329 & -3.436271 & -0.000105 \\ \mathrm{H} & -2.200583 & -4.045962 & -0.000636 \\ \mathrm{H} & -0.462882 & -2.242701 & -0.000534\end{array}$

Compound $35^{-}$

B3LYP Energy $=-700.6788136$

Sum of electronic and zero-point Energies $=-700.511542$

Sum of electronic and thermal Enthalpies $=-700.497851$

Sum of electronic and thermal Free Energies $=-700.553985$

Cartesian coordinates

$\begin{array}{cccc}\mathrm{O} & -0.351016 & -0.586485 & 0.021398 \\ \mathrm{C} & -1.586450 & 0.070402 & -0.067190 \\ \mathrm{~N} & -1.382098 & 1.382318 & -0.212462 \\ \mathrm{~N} & -0.017718 & 1.584312 & -0.226419 \\ \mathrm{C} & 0.557415 & 0.421392 & -0.086752 \\ \mathrm{~N} & -2.646364 & -0.728567 & -0.067758 \\ \mathrm{C} & -3.899652 & -0.262755 & 0.183856 \\ \mathrm{C} & -4.990303 & -1.295468 & -0.128244 \\ \mathrm{C} & 1.978560 & 0.124257 & -0.047540 \\ \mathrm{O} & -4.243087 & 0.842998 & 0.631398 \\ \mathrm{H} & -4.579898 & -2.302826 & -0.236003 \\ \mathrm{H} & -5.751886 & -1.273937 & 0.658316 \\ \mathrm{H} & -5.482874 & -1.015559 & -1.068600 \\ \mathrm{C} & 2.444025 & -1.198275 & 0.090949 \\ \mathrm{C} & 3.813564 & -1.466651 & 0.124836 \\ \mathrm{C} & 4.745100 & -0.428763 & 0.022577 \\ \mathrm{C} & 4.287397 & 0.889496 & -0.114788 \\ \mathrm{C} & 2.923674 & 1.166782 & -0.149674 \\ \mathrm{H} & 1.722418 & -2.004519 & 0.170455 \\ \mathrm{H} & 4.153865 & -2.493860 & 0.232207\end{array}$




$\begin{array}{lrrr}\mathrm{H} & 5.810731 & -0.640746 & 0.049706 \\ \mathrm{H} & 5.001322 & 1.705863 & -0.194805 \\ \mathrm{H} & 2.562420 & 2.184541 & -0.255355\end{array}$

Compound 36

B3LYP Energy=-700.6614477

Sum of electronic and zero-point Energies $=-700.493800$

Sum of electronic and thermal Enthalpies $=-700.480320$

Sum of electronic and thermal Free Energies=-700.534893

Cartesian coordinates

$\begin{array}{lrrr}\mathrm{C} & -1.244928 & -3.567423 & 0.000120 \\ \mathrm{~N} & -0.210707 & -2.793785 & 0.000066 \\ \mathrm{C} & -0.769916 & -1.493765 & -0.000009 \\ \mathrm{~N} & -2.107503 & -1.506778 & 0.000082 \\ \mathrm{O} & -2.420238 & -2.924822 & 0.000131 \\ \mathrm{~N} & 0.077613 & -0.440138 & -0.000053 \\ \mathrm{C} & -0.382257 & 0.825663 & -0.000030 \\ \mathrm{C} & 0.740292 & 1.861545 & -0.000067 \\ \mathrm{C} & -1.270721 & -5.060774 & 0.000167 \\ \mathrm{O} & -1.558780 & 1.244453 & 0.000051 \\ \mathrm{H} & -1.794824 & -5.441083 & 0.884320 \\ \mathrm{H} & -0.246572 & -5.437708 & 0.000363 \\ \mathrm{H} & -1.794493 & -5.441145 & -0.884159 \\ \mathrm{C} & 0.404583 & 3.223183 & -0.000031 \\ \mathrm{C} & 1.397042 & 4.207093 & -0.000064 \\ \mathrm{C} & 2.748049 & 3.842399 & -0.000132 \\ \mathrm{C} & 3.093107 & 2.485419 & -0.000168 \\ \mathrm{C} & 2.097855 & 1.504104 & -0.000136 \\ \mathrm{H} & -0.650773 & 3.478446 & 0.000022 \\ \mathrm{H} & 1.118216 & 5.259091 & -0.000036 \\ \mathrm{H} & 3.523158 & 4.606024 & -0.000157 \\ \mathrm{H} & 4.141256 & 2.192413 & -0.000221 \\ \mathrm{H} & 2.341708 & 0.447156 & -0.000163\end{array}$

Compound 37

B3LYP Energy=-700.6253688

Sum of electronic and zero-point Energies $=-700.460587$

Sum of electronic and thermal Enthalpies $=-700.446011$

Sum of electronic and thermal Free Energies=-700.503793

Cartesian coordinates

$\begin{array}{rrrr}\mathrm{C} & -1.917379 & 0.265298 & 0.240970 \\ \mathrm{C} & -0.825464 & -0.776009 & 0.066195 \\ \mathrm{O} & -1.081326 & -1.962093 & 0.329418 \\ \mathrm{~N} & 0.352843 & -0.257236 & -0.385521\end{array}$




$\begin{array}{lrrr}\mathrm{C} & 1.361471 & -1.094895 & -0.552652 \\ \mathrm{~N} & 2.683149 & -0.909896 & -1.082566 \\ \mathrm{C} & 3.699559 & -0.389355 & -0.270701 \\ \mathrm{C} & 4.115122 & 1.002755 & -0.735307 \\ \mathrm{~N} & 1.928677 & -2.228493 & -0.469700 \\ \mathrm{O} & 4.282301 & -0.977138 & 0.629601 \\ \mathrm{H} & 3.232691 & 1.637687 & -0.866421 \\ \mathrm{H} & 4.798065 & 1.441242 & -0.004282 \\ \mathrm{H} & 4.616730 & 0.935037 & -1.707117 \\ \mathrm{C} & -1.713543 & 1.622558 & -0.049730 \\ \mathrm{C} & -2.745666 & 2.548420 & 0.124922 \\ \mathrm{C} & -3.996014 & 2.130220 & 0.593621 \\ \mathrm{C} & -4.206227 & 0.777910 & 0.885770 \\ \mathrm{C} & -3.173491 & -0.145613 & 0.709780 \\ \mathrm{H} & -0.738168 & 1.927355 & -0.412177 \\ \mathrm{H} & -2.574799 & 3.597947 & -0.104127 \\ \mathrm{H} & -4.798873 & 2.851202 & 0.730036 \\ \mathrm{H} & -5.175278 & 0.444892 & 1.250686 \\ \mathrm{H} & -3.311050 & -1.199883 & 0.928550\end{array}$

Compound 37,

B3LYP Energy=-700.6279067

Sum of electronic and zero-point Energies $=-700.462863$

Sum of electronic and thermal Enthalpies $=-700.448501$

Sum of electronic and thermal Free Energies $=-700.505225$

Cartesian coordinates

$\begin{array}{lrrr}\mathrm{C} & -1.783843 & 0.130585 & 0.278493 \\ \mathrm{C} & -0.296247 & -0.092603 & 0.069453 \\ \mathrm{O} & 0.162603 & -1.237028 & 0.255442 \\ \mathrm{~N} & 0.363595 & 1.020630 & -0.333072 \\ \mathrm{C} & 1.683245 & 1.028770 & -0.409731 \\ \mathrm{~N} & 2.850335 & 0.355971 & 0.117031 \\ \mathrm{C} & 3.375898 & -0.768027 & -0.555695 \\ \mathrm{C} & 3.330125 & -2.006674 & 0.321766 \\ \mathrm{~N} & 2.689831 & 1.666341 & -0.839167 \\ \mathrm{O} & 3.894857 & -0.754089 & -1.662632 \\ \mathrm{H} & 2.287964 & -2.201737 & 0.593190 \\ \mathrm{H} & 3.755634 & -2.852396 & -0.223200 \\ \mathrm{H} & 3.895532 & -1.835167 & 1.244271 \\ \mathrm{C} & -2.388450 & 1.380814 & 0.078676 \\ \mathrm{C} & -3.759816 & 1.547222 & 0.288441 \\ \mathrm{C} & -4.546212 & 0.466213 & 0.702300 \\ \mathrm{C} & -3.949850 & -0.783525 & 0.903282 \\ \mathrm{C} & -2.579051 & -0.947859 & 0.691891 \\ \mathrm{H} & -1.762741 & 2.206324 & -0.242558 \\ \mathrm{H} & -4.216342 & 2.521538 & 0.129224 \\ \mathrm{H} & -5.613616 & 0.596318 & 0.865781 \\ \mathrm{H} & -4.554218 & -1.628961 & 1.224420\end{array}$


$\mathrm{H} \quad-2.093011 \quad-1.907054 \quad 0.839594$

Compound 38

B3LYP Energy $=-700.6568287$

Sum of electronic and zero-point Energies $=-700.491291$

Sum of electronic and thermal Enthalpies $=-700.476466$

Sum of electronic and thermal Free Energies=-700.535446

Cartesian coordinates

$\begin{array}{rrrr}\mathrm{C} & 2.388256 & -0.186217 & 0.018151 \\ \mathrm{C} & 0.989151 & -0.759760 & 0.019644 \\ \mathrm{O} & 0.829003 & -1.991572 & 0.040611 \\ \mathrm{~N} & 0.013716 & 0.191499 & -0.003361 \\ \mathrm{C} & -1.237652 & -0.146426 & -0.005163 \\ \mathrm{~N} & -2.402399 & -0.257110 & -0.010412 \\ \mathrm{~N} & -3.673360 & -0.674341 & -0.010938 \\ \mathrm{C} & -4.561624 & 0.354890 & -0.034643 \\ \mathrm{C} & -6.005058 & -0.140566 & -0.034756 \\ \mathrm{O} & -4.320796 & 1.577100 & -0.054762 \\ \mathrm{H} & -6.064738 & -1.231772 & -0.016362 \\ \mathrm{H} & -6.525403 & 0.270816 & 0.837804 \\ \mathrm{H} & -6.514878 & 0.240570 & -0.927032 \\ \mathrm{C} & 2.633847 & 1.195243 & -0.005022 \\ \mathrm{C} & 3.943283 & 1.681553 & -0.005542 \\ \mathrm{C} & 5.024988 & 0.793995 & 0.017094 \\ \mathrm{C} & 4.786720 & -0.584504 & 0.040267 \\ \mathrm{C} & 3.477275 & -1.069572 & 0.040758 \\ \mathrm{H} & 1.785601 & 1.870908 & -0.022402 \\ \mathrm{H} & 4.120401 & 2.754290 & -0.023631 \\ \mathrm{H} & 6.044153 & 1.173356 & 0.016674 \\ \mathrm{H} & 5.622156 & -1.280536 & 0.057944 \\ \mathrm{H} & 3.266226 & -2.134003 & 0.058471\end{array}$

Compound 39

B3LYP Energy= $=700.6227033$

Sum of electronic and zero-point Energies $=-700.457910$

Sum of electronic and thermal Enthalpies $=-700.443263$

Sum of electronic and thermal Free Energies $=-700.501611$

Cartesian coordinates
$\begin{array}{llll}\text { C } & -4.310440 & -0.117811 & 0.323033\end{array}$
$\begin{array}{llll}\text { C } & -3.205035 & -1.162849 & 0.473596\end{array}$
$\begin{array}{lllll}\mathrm{O} & -3.459739 & -2.271291 & 0.962057\end{array}$
$\begin{array}{llll}\mathrm{N} & -1.991707 & -0.712141 & 0.017562\end{array}$
$\begin{array}{llll}\text { C } & -0.959343 & -1.524991 & 0.111094\end{array}$
$\begin{array}{llll}\mathrm{N} & 0.395709 & -1.412573 & -0.332170\end{array}$ 


$\begin{array}{lrrr}\mathrm{C} & 1.409006 & -0.748977 & 0.346260 \\ \mathrm{C} & 1.679651 & 0.639182 & -0.191216 \\ \mathrm{~N} & -0.362731 & -2.601543 & 0.450513 \\ \mathrm{O} & 2.129708 & -1.241001 & 1.211274 \\ \mathrm{H} & -5.261245 & -0.528581 & 0.669694 \\ \mathrm{H} & -4.396935 & 0.193343 & -0.724160 \\ \mathrm{H} & -4.059742 & 0.777739 & 0.903220 \\ \mathrm{C} & 2.889912 & 1.264380 & 0.142041 \\ \mathrm{C} & 3.166343 & 2.557501 & -0.304722 \\ \mathrm{C} & 2.225945 & 3.246020 & -1.079508 \\ \mathrm{C} & 1.010501 & 2.632829 & -1.401031 \\ \mathrm{C} & 0.737491 & 1.333221 & -0.966062 \\ \mathrm{H} & 3.596051 & 0.716098 & 0.757790 \\ \mathrm{H} & 4.110922 & 3.030623 & -0.046675 \\ \mathrm{H} & 2.437237 & 4.255105 & -1.425749 \\ \mathrm{H} & 0.271138 & 3.167734 & -1.991954 \\ \mathrm{H} & -0.210265 & 0.859045 & -1.201071\end{array}$

Compound 39'

B3LYP Energy=-700.6230338

Sum of electronic and zero-point Energies $=-700.458074$

Sum of electronic and thermal Enthalpies $=-700.443636$

Sum of electronic and thermal Free Energies $=-700.500624$

Cartesian coordinates

$\begin{array}{lrrr}\mathrm{C} & 3.557117 & -1.896413 & 1.001892 \\ \mathrm{C} & 2.638689 & -1.054592 & 0.115969 \\ \mathrm{O} & 1.905380 & -1.637461 & -0.703422 \\ \mathrm{~N} & 2.778558 & 0.283590 & 0.309510 \\ \mathrm{C} & 1.969730 & 1.155485 & -0.256614 \\ \mathrm{~N} & 0.624718 & 1.292116 & -0.795573 \\ \mathrm{C} & -0.497003 & 1.523190 & 0.007908 \\ \mathrm{C} & -1.507915 & 0.402314 & -0.030507 \\ \mathrm{~N} & 1.798825 & 2.372154 & -0.569906 \\ \mathrm{O} & -0.738104 & 2.571120 & 0.601602 \\ \mathrm{H} & 4.057485 & -1.291864 & 1.761619 \\ \mathrm{H} & 4.312084 & -2.383296 & 0.372806 \\ \mathrm{H} & 2.969817 & -2.689556 & 1.476593 \\ \mathrm{C} & -2.770959 & 0.628141 & 0.538120 \\ \mathrm{C} & -3.734617 & -0.381021 & 0.546607 \\ \mathrm{C} & -3.439902 & -1.632896 & -0.006414 \\ \mathrm{C} & -2.178279 & -1.864947 & -0.563528 \\ \mathrm{C} & -1.213065 & -0.853741 & -0.583796 \\ \mathrm{H} & -2.971570 & 1.604312 & 0.968442 \\ \mathrm{H} & -4.712339 & -0.194553 & 0.985063 \\ \mathrm{H} & -4.187771 & -2.422804 & 0.002090 \\ \mathrm{H} & -1.938131 & -2.838705 & -0.982796 \\ \mathrm{H} & -0.225264 & -1.040951 & -0.995306\end{array}$




\section{Compound 40}

B3LYP Energy=-700.6568173

Sum of electronic and zero-point Energies $=-700.491606$

Sum of electronic and thermal Enthalpies $=-700.476543$

Sum of electronic and thermal Free Energies=-700.537338

Cartesian coordinates

$\begin{array}{lccc}\mathrm{C} & -4.744442 & -3.549980 & -1.111127 \\ \mathrm{C} & -3.298876 & -3.099678 & -0.930426 \\ \mathrm{O} & -2.370341 & -3.901674 & -1.086426 \\ \mathrm{~N} & -3.194264 & -1.782873 & -0.558386 \\ \mathrm{C} & -2.031891 & -1.251861 & -0.352969 \\ \mathrm{~N} & -1.087721 & -0.577403 & -0.175663 \\ \mathrm{~N} & 0.045588 & -0.106309 & 0.361284 \\ \mathrm{C} & 0.452743 & 1.053021 & -0.209496 \\ \mathrm{C} & 1.738757 & 1.570883 & 0.404741 \\ \mathrm{O} & -0.094816 & 1.697166 & -1.126954 \\ \mathrm{H} & -5.376195 & -2.732000 & -1.467470 \\ \mathrm{H} & -5.139743 & -3.880707 & -0.142359 \\ \mathrm{H} & -4.781147 & -4.395893 & -1.801711 \\ \mathrm{C} & 2.262901 & 2.779117 & -0.076047 \\ \mathrm{C} & 3.445967 & 3.305209 & 0.449115 \\ \mathrm{C} & 4.125505 & 2.627542 & 1.466519 \\ \mathrm{C} & 3.609602 & 1.420118 & 1.952314 \\ \mathrm{C} & 2.426359 & 0.895903 & 1.426754 \\ \mathrm{H} & 1.717490 & 3.284224 & -0.867037 \\ \mathrm{H} & 3.838771 & 4.243828 & 0.064257 \\ \mathrm{H} & 5.047216 & 3.034205 & 1.876570 \\ \mathrm{H} & 4.131771 & 0.885526 & 2.742770 \\ \mathrm{H} & 2.018121 & -0.038672 & 1.795109\end{array}$

\section{Transition States}

Transition State $\left[\mathbf{3 3}^{-} \rightarrow \mathbf{3 6}^{-}\right]^{\ddagger}$

B3LYP Energy=-700.6349288

Sum of electronic and zero-point Energies $=-700.468669$

Sum of electronic and thermal Enthalpies $=-700.455653$

Sum of electronic and thermal Free Energies=-700.508374

Cartesian coordinates

$\begin{array}{lrrr}\mathrm{C} & -3.817400 & -0.177246 & 0.000058 \\ \mathrm{~N} & -2.713620 & -0.905922 & 0.000050 \\ \mathrm{C} & -1.627410 & -0.046179 & 0.000037 \\ \mathrm{~N} & -1.779988 & 1.282161 & 0.000086 \\ \mathrm{O} & -3.780301 & 1.096379 & 0.000075 \\ \mathrm{~N} & -0.365473 & -0.619393 & 0.000012\end{array}$




$\begin{array}{lrcr}\mathrm{C} & 0.522872 & 0.357103 & 0.000000 \\ \mathrm{C} & 1.981012 & 0.020665 & -0.000043 \\ \mathrm{C} & -5.163812 & -0.873380 & 0.000144 \\ \mathrm{O} & 0.161907 & 1.583768 & -0.000012 \\ \mathrm{H} & -5.737887 & -0.570199 & 0.883068 \\ \mathrm{H} & -5.038251 & -1.958125 & -0.000182 \\ \mathrm{H} & -5.738270 & -0.569683 & -0.882350 \\ \mathrm{C} & 2.937631 & 1.046497 & -0.000079 \\ \mathrm{C} & 4.301753 & 0.744206 & -0.000118 \\ \mathrm{C} & 4.726832 & -0.588206 & -0.000124 \\ \mathrm{C} & 3.776159 & -1.616271 & -0.000090 \\ \mathrm{C} & 2.413166 & -1.315378 & -0.000049 \\ \mathrm{H} & 2.589133 & 2.074310 & -0.000074 \\ \mathrm{H} & 5.033476 & 1.548781 & -0.000145 \\ \mathrm{H} & 5.788421 & -0.824281 & -0.000156 \\ \mathrm{H} & 4.099330 & -2.654717 & -0.000094 \\ \mathrm{H} & 1.659941 & -2.096047 & -0.000021\end{array}$

Transition State $\left[\mathbf{3 3}^{-} \rightarrow \mathbf{3 7}\right]^{\ddagger}$

B3LYP Energy $=-700.6152534$

Sum of electronic and zero-point Energies $=-700.450855$

Sum of electronic and thermal Enthalpies $=-700.436916$

Sum of electronic and thermal Free Energies $=-700.492867$

Cartesian coordinates

$\begin{array}{crrc}\mathrm{C} & 3.673807 & 0.509096 & 0.395830 \\ \mathrm{~N} & 2.891906 & 0.329086 & -0.730993 \\ \mathrm{C} & 1.616529 & -0.225648 & -0.520578 \\ \mathrm{~N} & 1.976638 & -1.429424 & -0.706082 \\ \mathrm{O} & 3.448964 & 0.126791 & 1.546262 \\ \mathrm{~N} & 0.421865 & 0.357173 & -0.292898 \\ \mathrm{C} & -0.510387 & -0.615989 & -0.290954 \\ \mathrm{C} & -1.937301 & -0.183832 & -0.073494 \\ \mathrm{C} & 4.948485 & 1.287163 & 0.069741 \\ \mathrm{O} & -0.260536 & -1.840236 & -0.465169 \\ \mathrm{H} & 5.468720 & 1.546724 & 0.994381 \\ \mathrm{H} & 4.714987 & 2.191461 & -0.501584 \\ \mathrm{H} & 5.599671 & 0.667985 & -0.558183 \\ \mathrm{C} & -2.958980 & -1.144168 & -0.081541 \\ \mathrm{C} & -4.288859 & -0.766490 & 0.117020 \\ \mathrm{C} & -4.613308 & 0.577996 & 0.328064 \\ \mathrm{C} & -3.597435 & 1.540720 & 0.338587 \\ \mathrm{C} & -2.267741 & 1.163137 & 0.139163 \\ \mathrm{H} & -2.683570 & -2.181157 & -0.244984 \\ \mathrm{H} & -5.072874 & -1.520221 & 0.108523 \\ \mathrm{H} & -5.648379 & 0.873116 & 0.484006 \\ \mathrm{H} & -3.842612 & 2.587341 & 0.503687 \\ \mathrm{H} & -1.465099 & 1.892558 & 0.144198\end{array}$


Transition State $\left[\mathbf{3 3}^{-} \rightarrow \mathbf{3 9}\right]^{\ddagger}$

B3LYP Energy $=-700.5903034$

Sum of electronic and zero-point Energies $=-700.427573$

Sum of electronic and thermal Enthalpies $=-700.413379$

Sum of electronic and thermal Free Energies=-700.470104

Cartesian coordinates

$\begin{array}{lrrr}\mathrm{C} & 3.824729 & 0.065269 & -1.006048 \\ \mathrm{~N} & 2.480034 & 0.315287 & -0.976042 \\ \mathrm{C} & 1.747538 & 0.024901 & 0.115282 \\ \mathrm{~N} & 1.987716 & -0.439328 & 1.275706 \\ \mathrm{O} & 4.496919 & 0.298017 & -2.022149 \\ \mathrm{~N} & 0.365835 & 0.316792 & 0.085867 \\ \mathrm{C} & -0.567801 & -0.251840 & 0.846447 \\ \mathrm{C} & -1.980035 & 0.013872 & 0.342659 \\ \mathrm{C} & 4.536330 & -0.508056 & 0.227234 \\ \mathrm{O} & -0.423277 & -0.933494 & 1.885842 \\ \mathrm{H} & 5.585137 & -0.701301 & -0.011357 \\ \mathrm{H} & 4.055031 & -1.437644 & 0.557523 \\ \mathrm{H} & 4.477495 & 0.197592 & 1.064922 \\ \mathrm{C} & -3.057886 & -0.497155 & 1.078823 \\ \mathrm{C} & -4.371737 & -0.287502 & 0.653506 \\ \mathrm{C} & -4.624697 & 0.439316 & -0.514380 \\ \mathrm{C} & -3.553419 & 0.953335 & -1.253074 \\ \mathrm{C} & -2.239357 & 0.742373 & -0.828878 \\ \mathrm{H} & -2.836462 & -1.057076 & 1.981416 \\ \mathrm{H} & -5.198462 & -0.690232 & 1.234019 \\ \mathrm{H} & -5.647087 & 0.603665 & -0.846385 \\ \mathrm{H} & -3.740905 & 1.518158 & -2.163019 \\ \mathrm{H} & -1.400962 & 1.134331 & -1.394800\end{array}$

Transition State $\left[\mathbf{3 6}^{-} \rightarrow \mathbf{3 7}\right]^{*}$

B3LYP Energy $=-700.5839078$

Sum of electronic and zero-point Energies $=-700.421227$

Sum of electronic and thermal Enthalpies $=-700.406870$

Sum of electronic and thermal Free Energies $=-700.464205$

Cartesian coordinates

$\begin{array}{rrrr}\mathrm{C} & -2.519436 & -1.525726 & 1.619766 \\ \mathrm{~N} & -2.467198 & -0.513185 & 0.758159 \\ \mathrm{C} & -1.318031 & 0.267128 & 0.470630 \\ \mathrm{~N} & -0.563842 & 0.203991 & 1.488249 \\ \mathrm{O} & -1.713243 & -1.838591 & 2.520833 \\ \mathrm{~N} & -1.295252 & 0.976336 & -0.669789 \\ \mathrm{C} & -0.156145 & 1.510826 & -1.183463 \\ \mathrm{C} & 1.202681 & 0.851042 & -0.964530 \\ \mathrm{C} & -3.783268 & -2.385482 & 1.439391 \\ \mathrm{O} & -0.177383 & 2.498391 & -1.936972\end{array}$




$\begin{array}{lrrr}\mathrm{H} & -4.329308 & -2.407492 & 2.387915 \\ \mathrm{H} & -3.471728 & -3.410101 & 1.211521 \\ \mathrm{H} & -4.435783 & -2.015429 & 0.646926 \\ \mathrm{C} & 2.344734 & 1.665308 & -0.945482 \\ \mathrm{C} & 3.616755 & 1.109679 & -0.798720 \\ \mathrm{C} & 3.768295 & -0.277842 & -0.698424 \\ \mathrm{C} & 2.638027 & -1.099131 & -0.739800 \\ \mathrm{C} & 1.364358 & -0.538130 & -0.863346 \\ \mathrm{H} & 2.212596 & 2.736657 & -1.059143 \\ \mathrm{H} & 4.490805 & 1.756412 & -0.770594 \\ \mathrm{H} & 4.758315 & -0.714550 & -0.590218 \\ \mathrm{H} & 2.745227 & -2.178356 & -0.665510 \\ \mathrm{H} & 0.491109 & -1.181557 & -0.884257\end{array}$

Transition State $\left[\mathbf{3 6}^{-} \rightarrow \mathbf{3 9}\right]^{+}$

B3LYP Energy=-700.6147497

Sum of electronic and zero-point Energies $=-700.450277$

Sum of electronic and thermal Enthalpies $=-700.436352$

Sum of electronic and thermal Free Energies=-700.492348

Cartesian coordinates

$\begin{array}{lrrr}\mathrm{C} & -3.824863 & -0.160825 & -0.013054 \\ \mathrm{~N} & -2.612087 & -0.578100 & -0.446173 \\ \mathrm{C} & -1.687504 & -0.146091 & 0.428048 \\ \mathrm{~N} & -1.697870 & 0.527682 & 1.504305 \\ \mathrm{O} & -4.018917 & 0.513655 & 1.028644 \\ \mathrm{~N} & -0.297790 & -0.382161 & 0.510071 \\ \mathrm{C} & 0.507154 & 0.522866 & -0.152193 \\ \mathrm{C} & 1.968844 & 0.116766 & -0.143167 \\ \mathrm{C} & -5.019113 & -0.555546 & -0.870836 \\ \mathrm{O} & 0.168479 & 1.559118 & -0.732036 \\ \mathrm{H} & -5.737871 & -1.114661 & -0.261477 \\ \mathrm{H} & -4.708114 & -1.159651 & -1.725680 \\ \mathrm{H} & -5.525430 & 0.349513 & -1.224749 \\ \mathrm{C} & 2.881153 & 0.874584 & -0.891603 \\ \mathrm{C} & 4.236879 & 0.542397 & -0.908282 \\ \mathrm{C} & 4.699323 & -0.554599 & -0.172305 \\ \mathrm{C} & 3.795384 & -1.314487 & 0.577500 \\ \mathrm{C} & 2.437789 & -0.983265 & 0.590753 \\ \mathrm{H} & 2.499102 & 1.723842 & -1.449219 \\ \mathrm{H} & 4.933945 & 1.137956 & -1.493243 \\ \mathrm{H} & 5.755435 & -0.814148 & -0.182653 \\ \mathrm{H} & 4.148578 & -2.166984 & 1.152968 \\ \mathrm{H} & 1.721808 & -1.560787 & 1.164591 \\ & & & \end{array}$

Transition State $[\mathbf{3 7} \rightarrow \mathbf{3 9}]^{\ddagger}$

B3LYP Energy=-700.5914283

Sum of electronic and zero-point Energies $=-700.428583$

Sum of electronic and thermal Enthalpies $=-700.413953$ 
Sum of electronic and thermal Free Energies=-700.473132 Cartesian coordinates

$\begin{array}{lrrr}\mathrm{C} & 4.376578 & -0.921572 & 1.494702 \\ \mathrm{C} & 3.580917 & -0.593442 & 0.229005 \\ \mathrm{O} & 3.811848 & -1.228903 & -0.809924 \\ \mathrm{~N} & 2.667139 & 0.404192 & 0.421145 \\ \mathrm{C} & 1.734052 & 0.688918 & -0.544318 \\ \mathrm{~N} & 0.440322 & 0.215264 & -0.572695 \\ \mathrm{C} & -0.576065 & 0.965765 & -0.052854 \\ \mathrm{C} & -1.894718 & 0.213689 & 0.000793 \\ \mathrm{~N} & 2.046455 & 1.445692 & -1.496611 \\ \mathrm{O} & -0.530769 & 2.133030 & 0.362248 \\ \mathrm{H} & 4.181670 & -1.963042 & 1.774078 \\ \mathrm{H} & 4.114353 & -0.263669 & 2.325844 \\ \mathrm{H} & 5.446628 & -0.836315 & 1.274890 \\ \mathrm{C} & -2.998242 & 0.833692 & 0.603954 \\ \mathrm{C} & -4.230063 & 0.180357 & 0.674701 \\ \mathrm{C} & -4.374421 & -1.104523 & 0.139009 \\ \mathrm{C} & -3.277966 & -1.728662 & -0.465438 \\ \mathrm{C} & -2.044788 & -1.074918 & -0.533116 \\ \mathrm{H} & -2.861689 & 1.831830 & 1.007784 \\ \mathrm{H} & -5.078283 & 0.671802 & 1.145708 \\ \mathrm{H} & -5.333381 & -1.614832 & 0.192101 \\ \mathrm{H} & -3.383400 & -2.726649 & -0.884275 \\ \mathrm{H} & -1.183638 & -1.544005 & -0.996235\end{array}$

Transition State $[\mathbf{3 7}, \rightarrow \mathbf{3 8}]^{\ddagger}$

B3LYP Energy=-700.6149363

Sum of electronic and zero-point Energies $=-700.451618$

Sum of electronic and thermal Enthalpies $=-700.437272$

Sum of electronic and thermal Free Energies=-700.494192

Cartesian coordinates

$\begin{array}{lrrc}\mathrm{C} & 1.855796 & -0.285834 & -0.479348 \\ \mathrm{C} & 0.416146 & 0.121347 & -0.259045 \\ \mathrm{O} & 0.114817 & 1.315871 & -0.197694 \\ \mathrm{~N} & -0.434430 & -0.945988 & -0.126084 \\ \mathrm{C} & -1.700576 & -0.859529 & 0.000181 \\ \mathrm{~N} & -2.872860 & -0.177529 & 1.236643 \\ \mathrm{C} & -3.443459 & 1.024321 & 0.915435 \\ \mathrm{C} & -3.493357 & 1.943085 & 2.141179 \\ \mathrm{~N} & -2.905224 & -1.117157 & -0.026937 \\ \mathrm{O} & -3.925485 & 1.401193 & -0.161395 \\ \mathrm{H} & -3.060687 & 1.474504 & 3.028687 \\ \mathrm{H} & -2.942237 & 2.860177 & 1.906841\end{array}$




$\begin{array}{rrrr}\mathrm{H} & -4.535366 & 2.218612 & 2.339208 \\ \mathrm{C} & 2.256589 & -1.629012 & -0.530061 \\ \mathrm{C} & 3.597302 & -1.959620 & -0.742327 \\ \mathrm{C} & 4.553431 & -0.951616 & -0.905784 \\ \mathrm{C} & 4.159560 & 0.390249 & -0.855619 \\ \mathrm{C} & 2.819681 & 0.719434 & -0.643698 \\ \mathrm{H} & 1.504358 & -2.399332 & -0.399582 \\ \mathrm{H} & 3.896515 & -3.004308 & -0.779371 \\ \mathrm{H} & 5.596824 & -1.209389 & -1.071129 \\ \mathrm{H} & 4.897585 & 1.178699 & -0.982108 \\ \mathrm{H} & 2.489274 & 1.752304 & -0.600673\end{array}$

Transition State $\left[\mathbf{3 9}^{\prime} \rightarrow \mathbf{4 0}\right]^{*}$

B3LYP Energy=-700.6139699

Sum of electronic and zero-point Energies $=-700.450803$

Sum of electronic and thermal Enthalpies $=-700.436320$

Sum of electronic and thermal Free Energies $=-700.494125$

Cartesian coordinates

$\begin{array}{crrr}\mathrm{C} & 2.071086 & 2.240815 & 0.981116 \\ \mathrm{~N} & 0.802908 & 2.763682 & 1.101910 \\ \mathrm{C} & -0.250136 & 2.063363 & 1.281676 \\ \mathrm{~N} & -1.386857 & 1.731066 & 1.641268 \\ \mathrm{O} & 2.377573 & 1.049384 & 1.000728 \\ \mathrm{~N} & -1.188426 & 0.869748 & 0.353841 \\ \mathrm{C} & -1.038496 & -0.446754 & 0.696224 \\ \mathrm{C} & -0.909291 & -1.335114 & -0.532483 \\ \mathrm{C} & 3.126496 & 3.321839 & 0.778218 \\ \mathrm{O} & -1.058715 & -0.962141 & 1.822855 \\ \mathrm{H} & 4.122953 & 2.879984 & 0.838659 \\ \mathrm{H} & 3.015581 & 4.114583 & 1.525364 \\ \mathrm{H} & 2.992985 & 3.784523 & -0.206806 \\ \mathrm{C} & -0.807741 & -2.719569 & -0.336922 \\ \mathrm{C} & -0.680995 & -3.587703 & -1.423762 \\ \mathrm{C} & -0.651685 & -3.081355 & -2.727364 \\ \mathrm{C} & -0.749552 & -1.700233 & -2.931319 \\ \mathrm{C} & -0.878805 & -0.833308 & -1.843343 \\ \mathrm{H} & -0.826571 & -3.086786 & 0.684293 \\ \mathrm{H} & -0.601362 & -4.659386 & -1.254473 \\ \mathrm{H} & -0.551249 & -3.754831 & -3.575655 \\ \mathrm{H} & -0.724831 & -1.297325 & -3.941478 \\ \mathrm{H} & -0.957020 & 0.237923 & -1.989960\end{array}$

Transition State $\left[\mathbf{3 8} \rightarrow \mathbf{3 4}^{-}\right]^{\text {* }}$

B3LYP Energy $=-700.652355$

Sum of electronic and zero-point Energies $=-700.486887$

Sum of electronic and thermal Enthalpies $=-700.473243$

Sum of electronic and thermal Free Energies $=-700.528357$

Cartesian coordinates 


$\begin{array}{rrrr}\mathrm{C} & -1.638127 & 0.155418 & -1.269322 \\ \mathrm{C} & -0.282805 & 0.588583 & -0.756003 \\ \mathrm{O} & 0.023818 & 1.783595 & -0.768437 \\ \mathrm{~N} & 0.499213 & -0.463156 & -0.330001 \\ \mathrm{C} & 1.648361 & -0.251537 & 0.195070 \\ \mathrm{~N} & 2.853608 & -0.243932 & 0.347205 \\ \mathrm{~N} & 3.661340 & -0.152036 & 1.463380 \\ \mathrm{C} & 2.891355 & -0.091970 & 2.568076 \\ \mathrm{C} & 3.673756 & 0.023147 & 3.866154 \\ \mathrm{O} & 1.634672 & -0.120024 & 2.587546 \\ \mathrm{H} & 4.750459 & 0.025491 & 3.677828 \\ \mathrm{H} & 3.389136 & 0.946822 & 4.383455 \\ \mathrm{H} & 3.416491 & -0.813918 & 4.525727 \\ \mathrm{C} & -2.055827 & -1.183147 & -1.237905 \\ \mathrm{C} & -3.317905 & -1.540849 & -1.719317 \\ \mathrm{C} & -4.176148 & -0.565639 & -2.237875 \\ \mathrm{C} & -3.764986 & 0.771796 & -2.270810 \\ \mathrm{C} & -2.504636 & 1.128215 & -1.788528 \\ \mathrm{H} & -1.378092 & -1.924871 & -0.830000 \\ \mathrm{H} & -3.632574 & -2.581208 & -1.688191 \\ \mathrm{H} & -5.158314 & -0.844879 & -2.612092 \\ \mathrm{H} & -4.428194 & 1.535029 & -2.670962 \\ \mathrm{H} & -2.164197 & 2.158722 & -1.799960\end{array}$

Transition State $\left[\mathbf{4 0} \rightarrow \mathbf{3 5}^{-}\right]^{*}$

B3LYP Energy $=-700.6536046$

Sum of electronic and zero-point Energies $=-700.488353$

Sum of electronic and thermal Enthalpies $=-700.474599$

Sum of electronic and thermal Free Energies $=-700.529933$

Cartesian coordinates

$\begin{array}{lrrr}\mathrm{C} & -4.003547 & 0.029435 & -3.437098 \\ \mathrm{C} & -2.602143 & 0.420151 & -2.988017 \\ \mathrm{O} & -2.210059 & 1.582802 & -3.068101 \\ \mathrm{~N} & -1.884993 & -0.666077 & -2.512104 \\ \mathrm{C} & -0.723270 & -0.525318 & -2.004804 \\ \mathrm{~N} & 0.494164 & -0.639352 & -1.900484 \\ \mathrm{~N} & 1.280994 & -0.518538 & -0.773682 \\ \mathrm{C} & 0.505807 & -0.251788 & 0.288432 \\ \mathrm{C} & 1.235362 & -0.084287 & 1.590860 \\ \mathrm{O} & -0.751905 & -0.138742 & 0.246826 \\ \mathrm{H} & -4.581474 & -0.309409 & -2.569563 \\ \mathrm{H} & -3.957362 & -0.810117 & -4.138874 \\ \mathrm{H} & -4.500194 & 0.884355 & -3.900101 \\ \mathrm{C} & 0.499872 & 0.201733 & 2.750639\end{array}$




$\begin{array}{lrrr}\mathrm{C} & 1.142144 & 0.361262 & 3.981698 \\ \mathrm{C} & 2.531836 & 0.236330 & 4.072108 \\ \mathrm{C} & 3.272759 & -0.049009 & 2.917907 \\ \mathrm{C} & 2.631797 & -0.207498 & 1.688215 \\ \mathrm{H} & -0.577396 & 0.294816 & 2.657991 \\ \mathrm{H} & 0.556998 & 0.583684 & 4.871397 \\ \mathrm{H} & 3.033416 & 0.359956 & 5.029241 \\ \mathrm{H} & 4.354542 & -0.147045 & 2.978215 \\ \mathrm{H} & 3.192332 & -0.427016 & 0.786137\end{array}$

NMR Spectrum of Compound 29b in DMSO- $d_{6}$ 


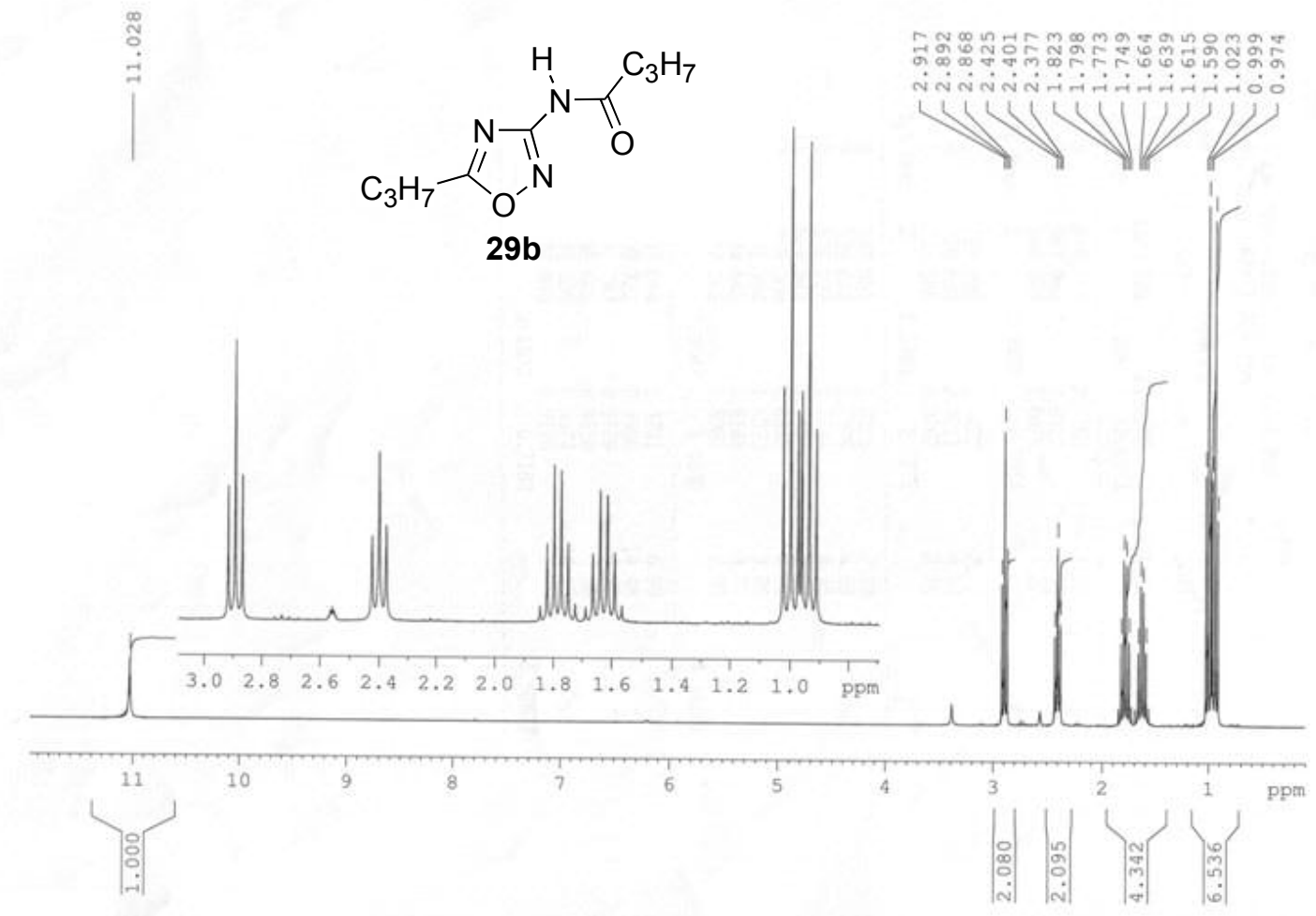

NMR Spectrum of Compound 29d in DMSO- $d_{6}$

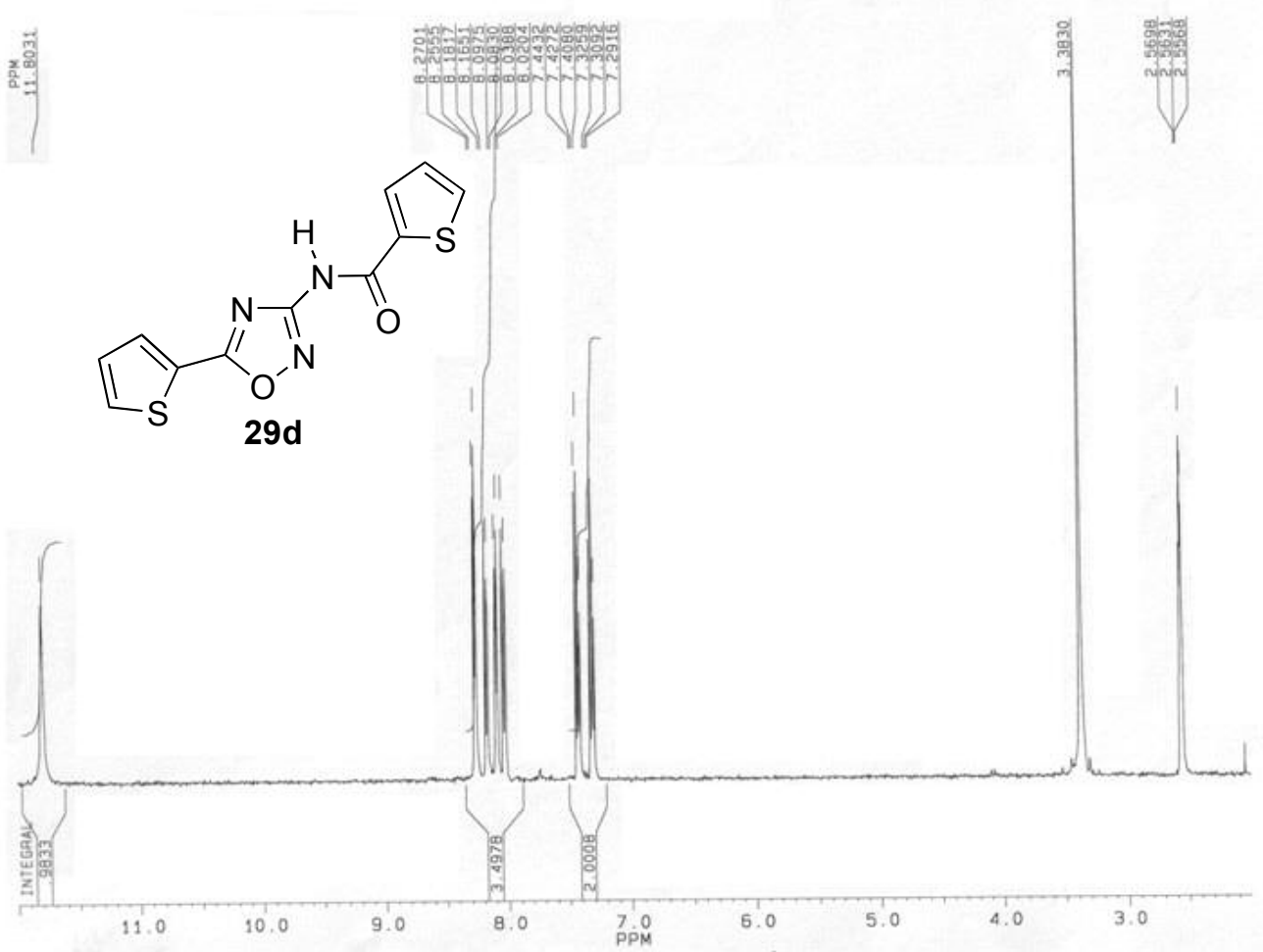

NMR Spectrum of Compound 30a in DMSO- $d_{6}$ 
$\stackrel{\mathrm{H}}{\mathrm{H}} \mathrm{C}-\mathrm{O}_{\mathrm{H}}^{\mathrm{N}-\mathrm{N}} \stackrel{\mathrm{O}}{\mathrm{N}} \stackrel{\mathrm{C}}{\mathrm{C}}-\mathrm{CH}_{3}$

$30 a$

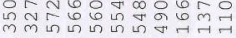

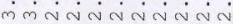

14.11/

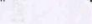
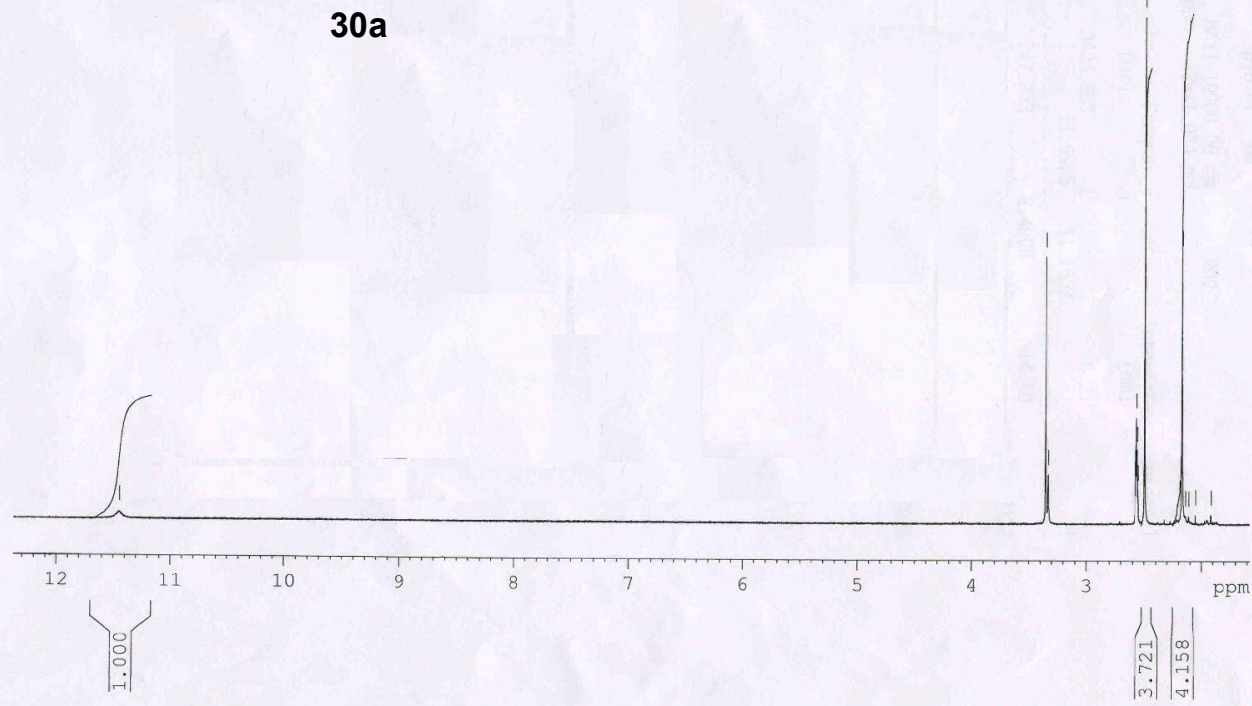

NMR Spectrum of Compound 30b in DMSO- $d_{6}$

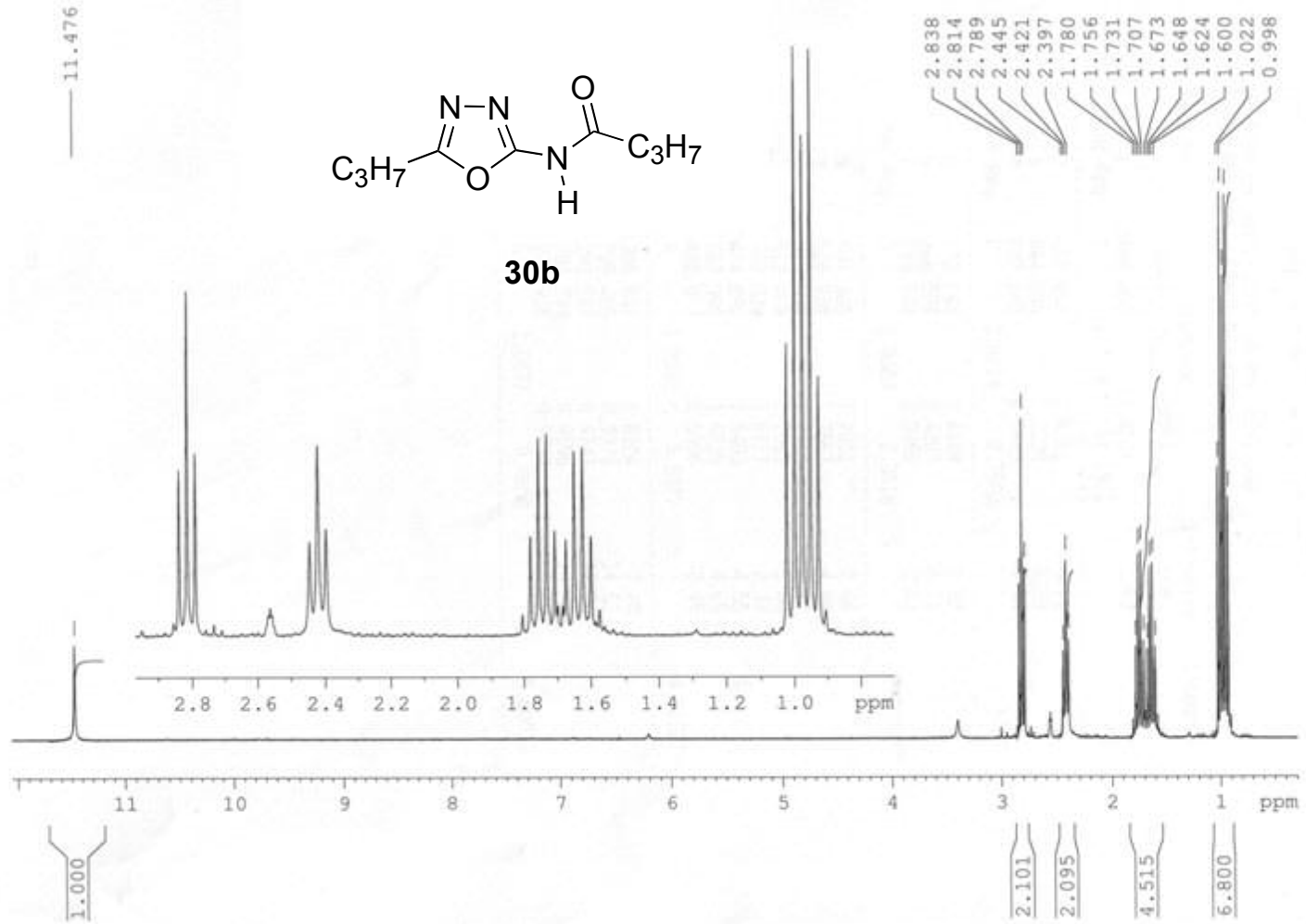

NMR Spectrum of Compound 30c in DMSO- $d_{6}$ 


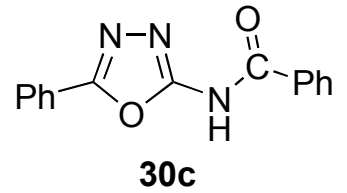

NMR Spectrum of Compound 30d in DMSO- $d_{6}$

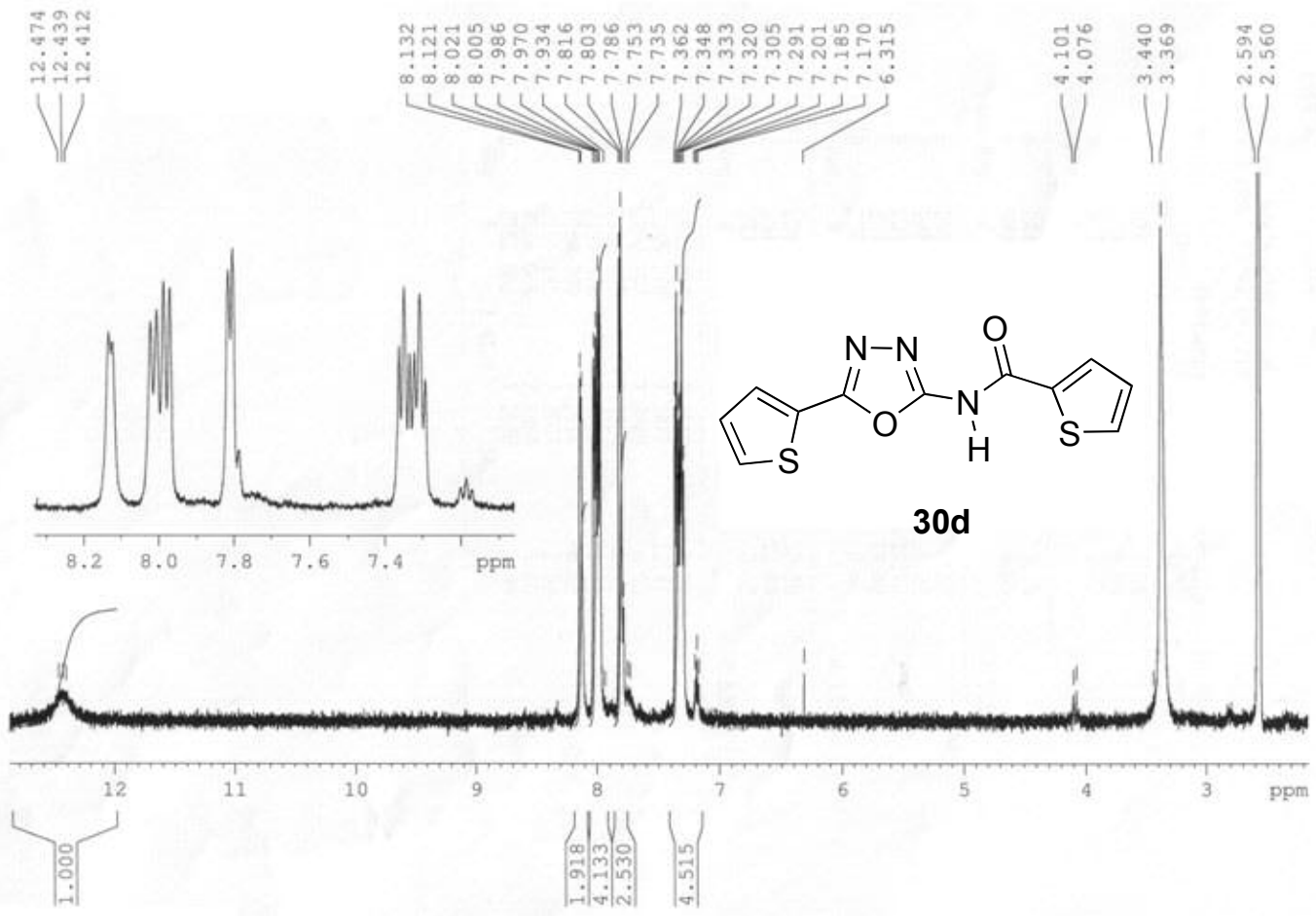

NMR Spectrum of Compound 34 in DMSO- $d_{6}$ 


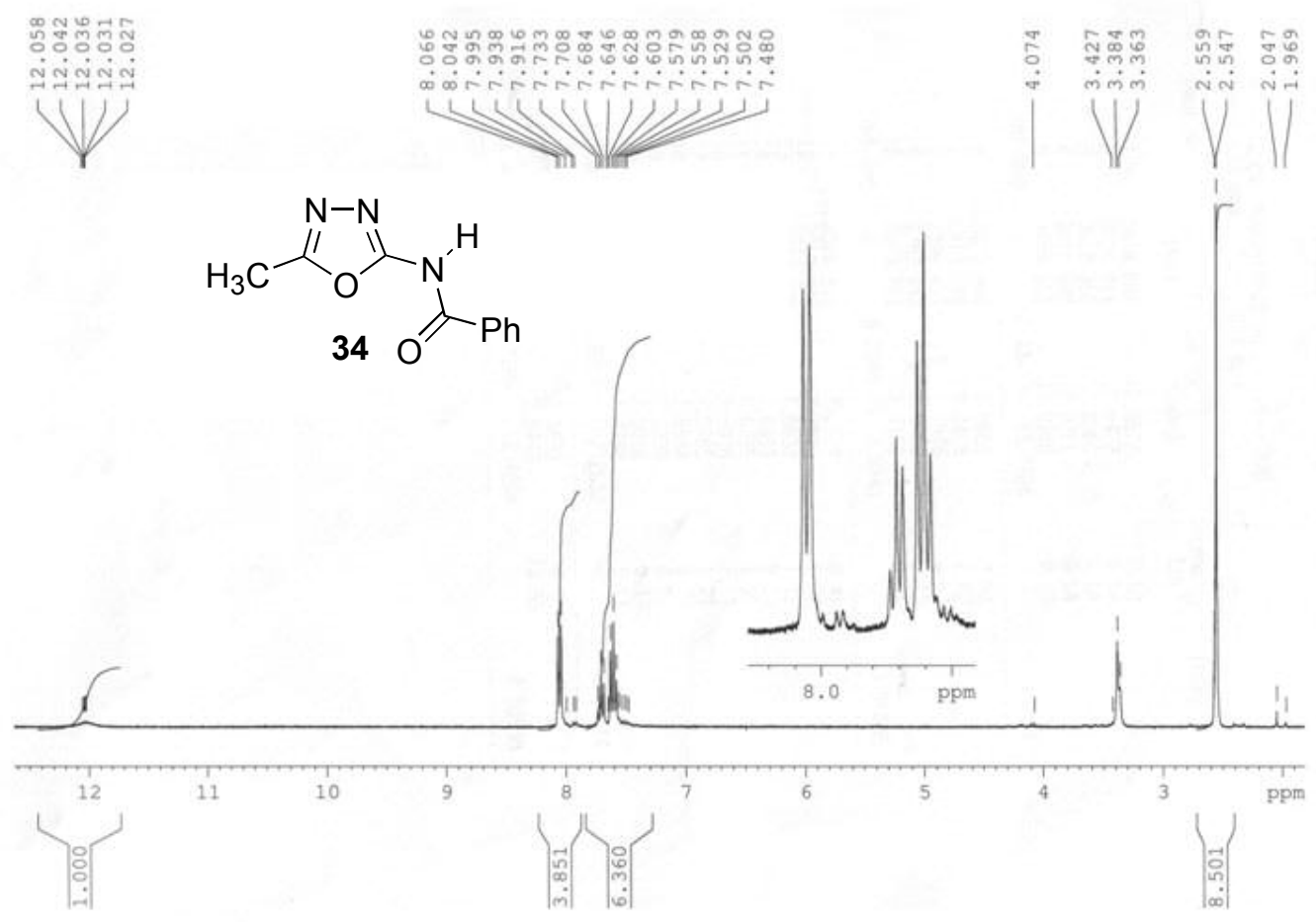

NMR Spectrum of Compound 35 in DMSO- $d_{6}$
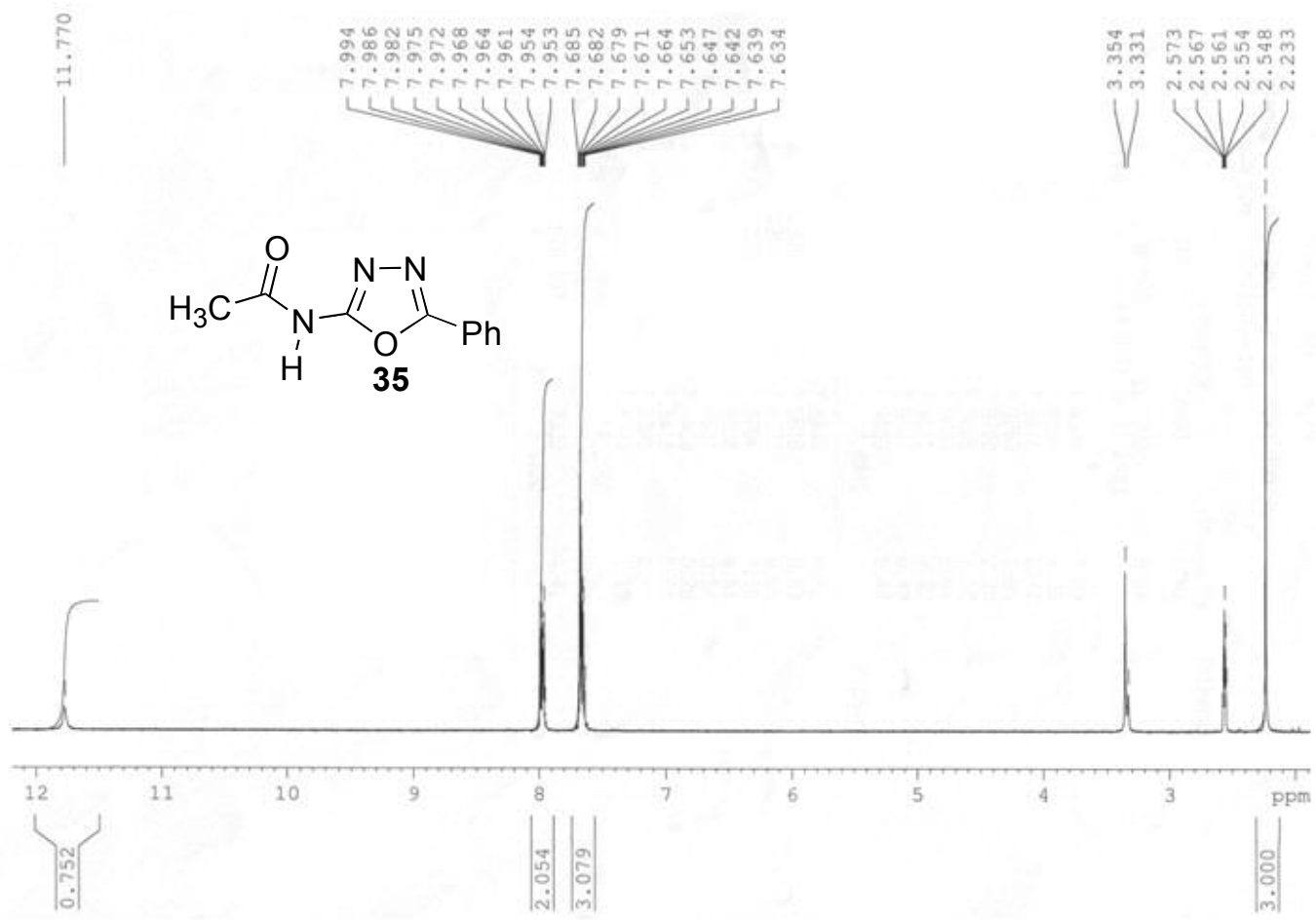